\title{
Mineralogy and defect microstructure of an olivine-dominated Itokawa dust particle: evidence for shock metamorphism, collisional fragmentation, and LL chondrite origin
}

\author{
Falko Langenhorst ${ }^{1 *}$, Dennis Harries ${ }^{1}$, Kilian Pollok ${ }^{1}$ and Peter A van Aken ${ }^{2}$
}

\begin{abstract}
We report here detailed analytical scanning and transmission electron microscopic investigations on an olivine-dominated dust particle (RB-QD04-0042) from the surface of asteroid 25143 Itokawa. The dust particle was returned to Earth by the Hayabusa spacecraft and was made available in the context of the first announcement of opportunity for Hayabusa sample investigation. Multiple thin slices were prepared from the precious particle by means of focused ion beam thinning, providing a unique three-dimensional access to its interior. The $40 \times 50 \mu \mathrm{m}$ sized olivine particle contains a spherical diopside inclusion and an intimate intergrowth of troilite and tetrataenite. The compositions of olivine $\left(\mathrm{FO}_{69} \mathrm{Fa}_{31}\right)$ and diopside $\left(\mathrm{En}_{48} \mathrm{WO}_{42} \mathrm{Fs}_{10}\right)$, as well as the high Ni content of the sulfide-metal alloy, indicate a LL ordinary chondrite origin in accord with previous classifications. Although no impact crater exists at the surface of RB-QD04-0042, transmission electron microscopy revealed the presence of various shock defects in constituent minerals. These defects are planar fractures and [001] screw dislocations in olivine, multiple $\{101\}$ deformation twins in tetrataenite and basal (0001) stacking faults in troilite. These diagnostic shock indicators occur only in a small zone on one concave side of the dust particle characterized by a high fracture density. These observations can be explained by a collisional event that spalled off material from the particle's surface. Alternatively, the dust particle itself could be a spallation fragment of an impact into a larger regolith target. This suggests that Itokawa dust particles lacking visible microcraters on their surfaces might have still experienced shock metamorphism and were involved in collisional fragmentation that resulted in the formation of regolith.
\end{abstract}

Keywords: Hayabusa; Itokawa; Shock metamorphism; Collisional fragmentation; Ordinary chondrite; Olivine; Tetrataenite; Troilite; Diopside

\section{Findings Introduction}

In June 2010, the Hayabusa space mission successfully returned precious dust particles from the rubble-pile S-type asteroid 25143 Itokawa to Earth. More than 1,500 particles with sizes in the range from 3 to $200 \mu \mathrm{m}$ were recovered, about one third of them representing olivinedominated aggregates (Nakamura et al. 2011). Access to these pristine materials provides for the first time direct

\footnotetext{
* Correspondence: Falko.Langenhorst@uni-jena.de

${ }^{1}$ Analytical Mineralogy of Micro- and Nanostructures, Institute of Geoscience, Friedrich Schiller University Jena, Carl-Zeiss-Promenade 10, D-07745 Jena, Germany

Full list of author information is available at the end of the article
}

information on surface processes (Tsuchiyama 2014), taking place on a primitive body in the solar system (space weathering, impact processes, regolith formation). Subsequent analyses on silicate minerals substantiate that the particles match the isotopic and mineralogic composition of $L L$ ordinary chondrites (Nakamura et al. 2011; Yurimoto et al. 2011; Nakashima et al. 2013) and reveal also that space weathering has affected a large portion of grains (Noguchi et al. 2011; Nakamura et al. 2012). Space weathering is attributed to the solar wind, which triggers the formation of $\mathrm{Fe}$ nanoparticles in amorphous surface layers of Fe-bearing silicates. This surface modification is the generally accepted cause for the observed alteration of 
the spectral colors of S-type chondrites (Kerr 2011; Noguchi et al. 2011).

In the first international announcement of opportunity for Hayabusa sample investigation, we received two olivinedominated particles (Abe et al. 2012): RA-QD02-0115 and RB-QD04-0042. We report here detailed scanning (SEM) and transmission electron microscopic (TEM) investigations of Hayabusa sample RB-QD04-0042, which was originally described to be mainly composed of olivine. Results on particle RA-QD02-0115 are presented in a companion paper (Harries and Langenhorst 2014).

In the course of focused ion beam (FIB) preparation and electron microscopic (EM) study, the olivine particle RB-QD04-0042 turned out to contain inclusions of diopside, troilite, and tetrataenite. The site-specific highresolution identification of these phases demonstrates that the combination of FIB-EM techniques is a powerful approach for the study of small extraterrestrial materials from sample-return missions.

Following FIB preparation, the mineralogical compositions and defect microstructures of constituent phases in RB-QD04-0042 were characterized in a TEM. The investigations aimed at verifying the ordinary chondrite signature and, in particular, at evaluating the shock damage of minerals. Previous studies report the existence of microcraters and fractures on the surfaces of Itokawa particles (Nakamura et al. 2012), but so far, little information is available on shock metamorphism of Hayabusa particles, although this process reflects the impact history of Itokawa, too, and thus provides hints to the more general process of regolith formation.

\section{Preparation and methods}

Mineral grains and morphology of particle RB-QD04-0042 were first inspected by means of scanning electron microscopy (Figure 1a) and energy-dispersive X-ray microanalysis (EDX) using the FEI Quanta3D FEG dual beam workstation (FEI Co., Eindhoven, NED) at the Institute of Geoscience, University of Jena, Germany. The machine is equipped with an EDAX/Ametek $\mathrm{Si}(\mathrm{Li})$ detector (EDAX, Mahwah, NJ, USA) and ultra-thin window (UTW), allowing the simultaneous detection of light and heavy elements.

After the first SEM observation, the workstation was employed for FIB preparation of TEM foils. For this purpose, a manually operated, vibration-damped tungsten needle was used to transfer the dust particle from the dimpled quartz glass plate to a polished 12- $\mathrm{mm}$ aluminum SEM stub that had been coated with a thin $(<10 \mu \mathrm{m})$ layer of glycol phthalate (GP). The stub with the grain resting on top of the GP layer was transferred into a glass vessel filled with acetone vapor and put to rest for $10 \mathrm{~min}$ to liquefy the GP layer. The latter formed a meniscus under the grain and fixed it securely to the stub. After the complete evaporation of any residual acetone, the sample stub was coated with carbon and inspected by SEM. At this point, a small adhering olivine grain (subsample RB-QD04-0042-01) was removed from the main grain and attached to a copper FIB-TEM grid by using the internal micromanipulator of the FIB-SEM workstation in combination with $\mathrm{Ga}^{+}$ion beam-assisted platinum deposition. After this, the surface of the main grain was covered with a platinum layer via electron beam-assisted deposition and deep trenches on three sides of the grain were milled into the aluminum stub. Undercutting of the grain left it suspended on a thin aluminum cantilever, which allowed cutting off a series of three 7- to 8- $\mu$ m-thick sections at predetermined locations (Figure 1b), which were protected by additional platinum layers.

The sections were attached to copper FIB-TEM grids, and two were thinned to electron transparency using successively lower $\mathrm{Ga}^{+}$ion beam currents of $5 \mathrm{nA}, 1 \mathrm{nA}$, and $300 \mathrm{pA}$ at $30-\mathrm{keV}$ energy (subsamples RB-QD040042-02 and RB-QD04-0042-03). Final polishing was done using a current of $150 \mathrm{pA}$ at $5-\mathrm{keV}$ ion energy. A third slice was preserved for future studies at a thickness of $3 \mu \mathrm{m}$ (RB-QD04-0042-04).

The two foils RB-QD04-0042-02 and RB-QD04-0042-03 were subsequently studied using the Philips CM200 transmission electron microscope (FEI Co.) at the Max Planck Institute for Intelligent Systems, Stuttgart, Germany. The microscope is equipped with an EDAX energy-dispersive system and a Gatan Erlangshen CCD camera (Gatan, Inc., Pleasanton, CA, USA), and operates with a $\mathrm{LaB}_{6}$ electron source at $200-\mathrm{kV}$ acceleration voltage. EDX analyses were quantified according to the Cliff-Lorimer technique involving an absorption correction and the use of calibration $k_{\mathrm{x} / \mathrm{Si}}$ factors for elements $\mathrm{X}$ with respect to $\mathrm{Si}$ (van Cappellen and Doukhan 1994; Langenhorst et al. 1995). Lattice defects were imaged and identified by combining selected area electron diffraction (SAED) and conventional brightfield and dark-field imaging techniques involving, in particular, the weak-beam imaging technique.

\section{Results}

\section{Mineralogy of particle RB-QD04-0042}

The original olivine grain RB-QD04-0042 is $40 \times 50 \mu \mathrm{m}$ in size and according to qualitative EDX analyses on the SEM shows an intimate $10-\mu \mathrm{m}$-wide intergrowth of FeNi alloy and iron sulfide at its surface (Figure 1a). Subsample RB-QD04-0042-02 is a FIB cut through this three-phase assemblage: olivine $+\mathrm{FeNi}$ alloy + iron sulfide (Figure 2a,c). In the course of FIB preparation, a rounded $6-\mu \mathrm{m}$-diameter diopside inclusion emerged deeper in the olivine particle (Figure 2b,d); this foil was labeled subsample RB-QD040042-03.

TEM-EDX spectra of all these phases were acquired and evaluated to yield quantitative compositional results. Olivine shows a fairly homogeneous composition throughout the 

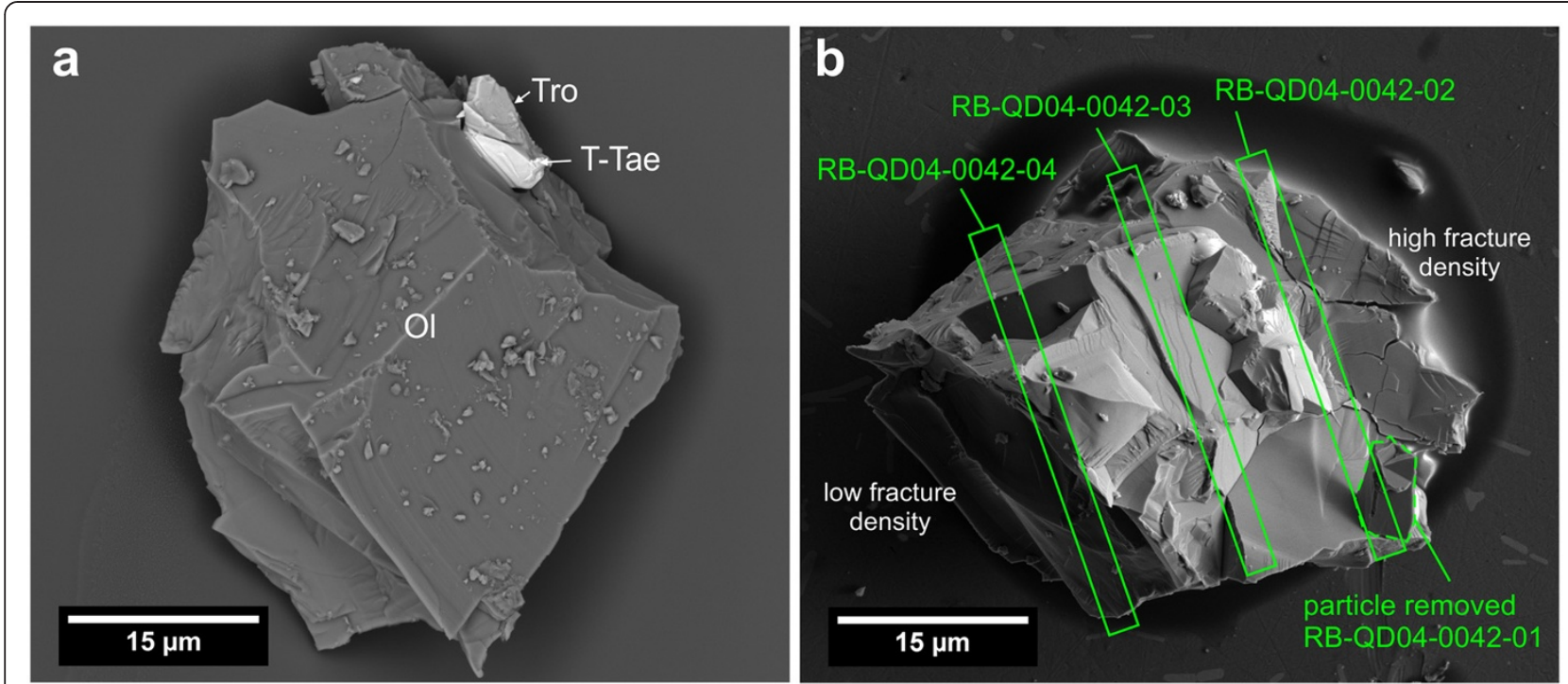

Figure 1 Scanning electron images of Hayabusa particle RB-QD04-0042. (a) Backscatter electron image of the particle as received, resting in a pit of the transport glass slide. The grain consists of olivine (Ol) with a troilite (Tro)-tetrataenite (T-Tae) aggregate on its upper right side (bright); fine-grained material on the surface represents loose olivine particles. (b) Secondary electron image of the particle after mounting on the Al stub. The locations of FIB sections are marked. Notably, the grain shows a highly fractured region on the right side.

particle (Figure 3a,b,c), ranging from $\mathrm{Fo}_{69} \mathrm{Fa}_{31}$ (RB-QD040042-02) to $\mathrm{Fo}_{68} \mathrm{Fa}_{32}$ (RB-QD04-0042-03). The composition of diopside is also uniform throughout the rounded grain and corresponds to $\mathrm{En}_{48} \mathrm{Wo}_{42} \mathrm{Fs}_{10}$.

The FeNi alloy shown in Figure 4a is rich in Ni and possesses the molar composition $\mathrm{Ni}_{51-52} \mathrm{Fe}_{44-45} \mathrm{Co}_{3-4}$. Electron diffraction patterns of this alloy reveal an ordered superstructure with tetragonal symmetry, being compatible with the tetrataenite structure, in which pure $\mathrm{Fe}$ and $\mathrm{Ni}$ layers alternate along the tetragonal $c$ axis (Clarke and Scott 1980; Uehara et al. 2011).

EDX analyses of iron sulfide point to a monosulfide composition but are not sufficiently precise to distinguish between troilite and the various pyrrhotite superstructures (Harries et al. 2011). Electron diffraction patterns of the monosulfide are however clearly in accord with the structure of the stoichiometric FeS phase, troilite (Figure 4d). It is noteworthy that the Ni $K$ peak is absent in the EDX spectra of troilite.

\section{Defect microstructures in minerals}

Defect microstructures are observed at the scale of tens of micrometers (i.e., at the grain size scale) down to the submicrometer level. At the grain scale, SEM images of subsample RB-QD04-0042-02 reveal several irregular fractures in olivine and along grain boundaries adjacent to the troilite-tetrataenite aggregate (Figures $1 \mathrm{~b}$ and $2 \mathrm{a}$ ). This finding is contrary to the appearance of subsample RB-QD04-0042-03, which is absolutely coherent and devoid of fractures (Figures $1 \mathrm{~b}$ and $2 \mathrm{~b}$ ). The difference in the mechanical state of the two subsamples is also obvious from the defect microstructures of individual minerals studied by TEM.

Olivine Olivine contains planar fractures and abundant straight dislocations throughout the entire subsample RBQD04-0042-02 (Figure 3a,b,c). Planar fractures occur as multiple sets of up to $5 \mu \mathrm{m}$ long and 1- to $2-\mu \mathrm{m}$ spaced closed fissures, extending from the surface of olivine to the grain boundary with tetrataenite (Figure 3a). The plane of the fractures can be indexed as $(0 \mathrm{kl})$ and is most likely (013) or (014), but an exact solution or view parallel to the fracture was not possible due to limited tilting range. Such prismatic fracture planes are typical for olivine in shocked ordinary chondrites (Langenhorst et al. 1995).

The dislocations are well visible in weak-beam images using the diffraction vector $g=$ [004] (Figure 3c) and are out of contrast with diffraction vectors perpendicular to the $c$ axis. The straight dislocation lines $l$ lie exactly along $l=[001]$. Thus, the dislocations possess Burgers vector $b=[001]$ and are of screw character. A determination of the glide plane was impossible due to the pure screw character. The density of [001] screw dislocations can be up to $10^{13} \mathrm{~m}^{-2}$ in subsample RB-QD04-0042-02. This type of dislocation occurs in both naturally and experimentally shocked olivine (Langenhorst et al. 1995, 1999, 2002) and can be clearly related to shock metamorphism.

Olivine in subsample RB-QD04-0042-03 displays a completely different microstructure. The section contains two high-angle boundaries separating three different olivine crystals (Figure 2d). Only the smallest 

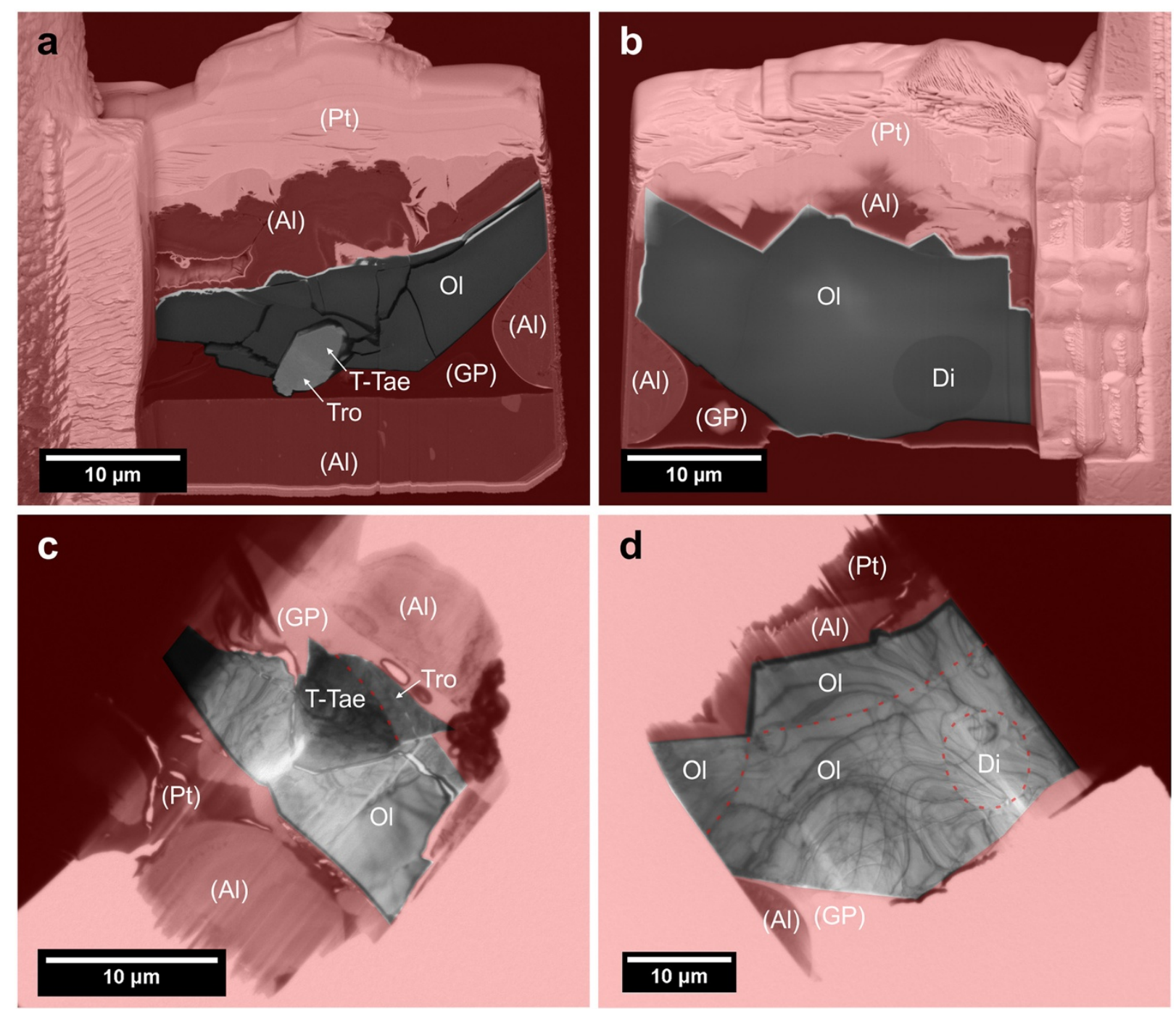

Figure 2 Backscatter electron (upper figures) and bright-field TEM (lower figures) images of the two complete subsections. (a, c) RB-QD04-0042-02 and (b, d) RB-QD04-0042-03. The greyish areas represent sample areas $(\mathrm{Ol}=$ olivine, Tro $=$ troilite, $\mathrm{T}-\mathrm{Tae}=$ tetrataenite, $\mathrm{Di}=$ diopside), while areas masked in red color are residues from the preparation process ( $\mathrm{GP}=$ glycol phthalate, $\mathrm{Pt}=$ platinum, $\mathrm{Al}=\mathrm{aluminum}$ ).

(approximately $8 \mu \mathrm{m}$ in diameter) triangular crystal in one corner of the section contains abundant [001] screw dislocations (Figures $2 \mathrm{~d}$ and 3c). The rest of the $25 \times 30$ $\mu \mathrm{m}$ large olivine section is virtually free of lattice defects.

Diopside A single crystal of diopside occurs as spherical inclusion in olivine. Since there is no epitaxial relationship between host and inclusion, diopside adapted this spherical shape due to the minimization of surface energy.

Diopside is generally a good shock indicator that can develop dislocations, mechanical twins, and even planar deformation features under dynamic compression (Leroux et al. 1994; Langenhorst et al. 1995; Jacob et al. 2009). However, the rounded diopside inclusion in subsample RB-QD04-0042-03 is devoid of lattice defects with the exception of a single dislocation (Figure 3d). This is in line with the finding that the surrounding host olivine is unshocked, too.

Tetrataenite Multiple sets of intersecting lamellae and abundant perfect dislocations are present in tetrataenite from subsample RB-QD04-0042-02 (Figure 4a). The nature of the lamellae is obvious from electron diffraction patterns, revealing a twin relation between lamellae and the bulk tetrataenite. For simplification, the electron diffraction pattern shown in Figure $4 \mathrm{~b}$ has been indexed in the face-centered cubic structure (fcc). The $[201]_{\text {fcc }}$ zone axis pattern shows a superposition of two lattice orientations, which are compatible with spinel-type $\{111\}$ twinning. In the tetragonal tetrataenite structure, the corresponding twin planes are indexed as $\{101\}$. The twin boundaries are decorated with abundant partial dislocations, attesting to 

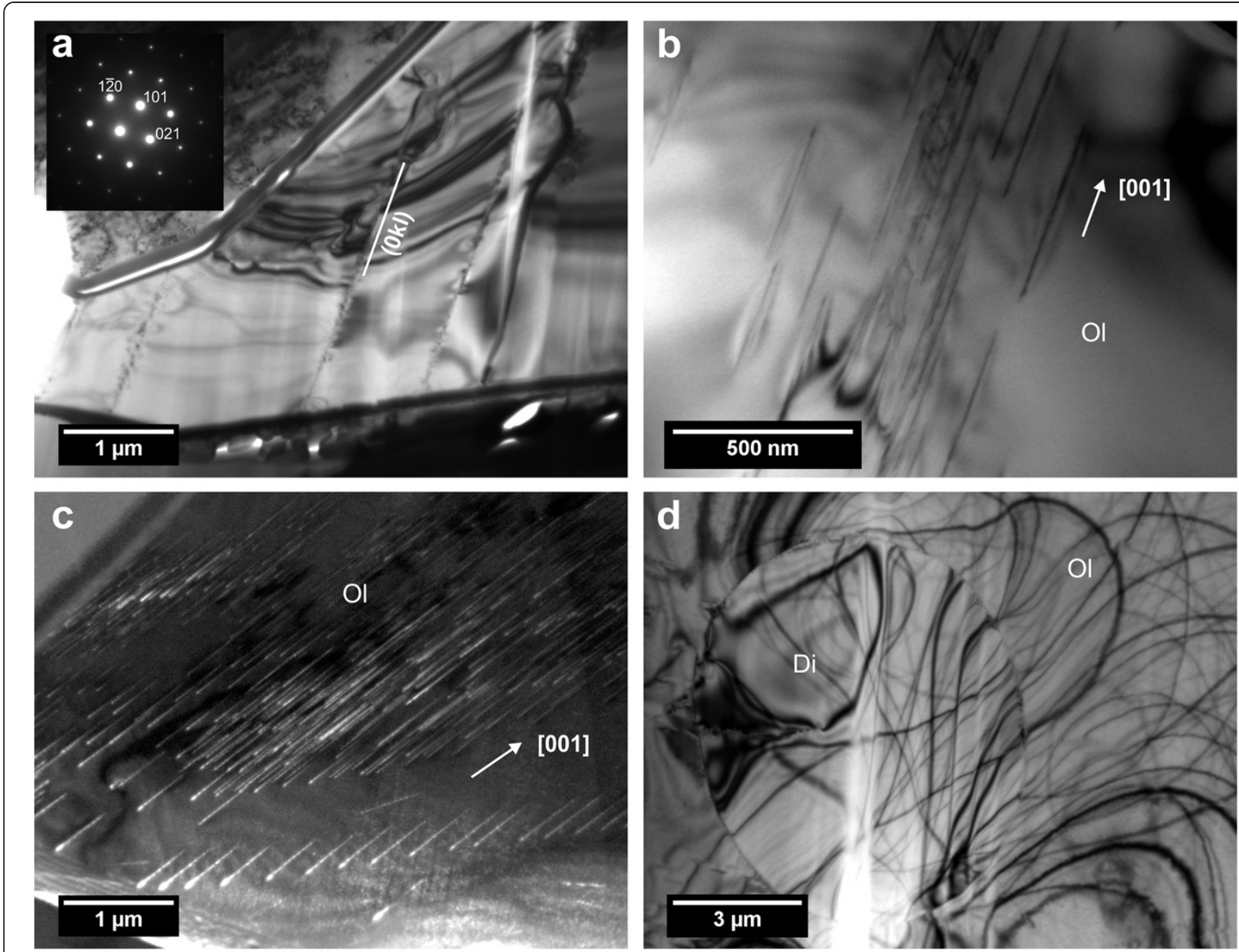

Figure 3 TEM images of silicate grains in RB-QD04-0042. (a) Planar fractures in olivine from subsample RB-QD04-0042-02. Based on two images with different zone axis orientations, the plane's orientation is $(0 \mathrm{kl})$, most likely (013) or (014). It is not viewed edge-on in this orientation. (b,c) The [001] screw dislocations in olivine (OI). (d) Defect-free spherical diopside (Di) as inclusion in olivine (subsample RB-QD04-0042-03). The manifold curved lines in (d) are bend contours.

the mechanical nature of the twins. Such shock twinning of tetrataenite has previously been reported for ordinary chondrites (Leroux 2001; Uehara et al. 2011).

Troilite Troilite contains several stacking faults parallel to the basal (0001) plane (Figure 4c,d). The faults are decorated with partial dislocations, demonstrating that they are produced by deformation. Similar types of stacking faults in pyrrhotite have been described for terrestrial impact rocks (Mang et al. 2012) and are regarded as shock indicator.

In addition to stacking faults, troilite is characterized by a recrystallization texture with numerous subgrains composing the entire grain.

\section{Discussion}

\section{Clues to chondritic origin and metamorphic cooling}

Previous mineralogical and isotopic studies have substantiated the link between the Hayabusa particles and equilibrated $L L$-type ordinary chondrites (Nakamura et al. 2011, 2012; Yurimoto et al. 2011; Nakashima et al. 2013). The mineral compositions determined in this study are in full agreement with this interpretation. The lines of support are as follows: (1) a Fa 31-32 content of olivine (range for $L L$ chondrites: Fa 26-33, Nakamura et al. 2011), (2) a Fs 10 content of diopside (mean value for type 4-6 $L L$ ordinary chondrite is Fs 10 , see Brearley and Jones 1998), and (3) the occurrence of a Ni-rich troilitetetrataenite aggregate with an $\mathrm{Fe} /(\mathrm{Fe}+\mathrm{Ni})$ ratio of 0.46 in the metal phase. The latter argument is based on the fact that the $\mathrm{Fe} /(\mathrm{Fe}+\mathrm{Ni})$ ratios in metal decrease in the following sequence (Jarosevich 1990): $H(0.91), L(0.86)$, and $L L$ ordinary chondrite $(0.71)$. It has however to be admitted that our argument is not very robust, because particle RBQD04-0042 contains only one sulfide-metal aggregate and the high $\mathrm{Ni}$ content of the aggregate considerably deviates from the average value for $L L$ ordinary chondrites. 


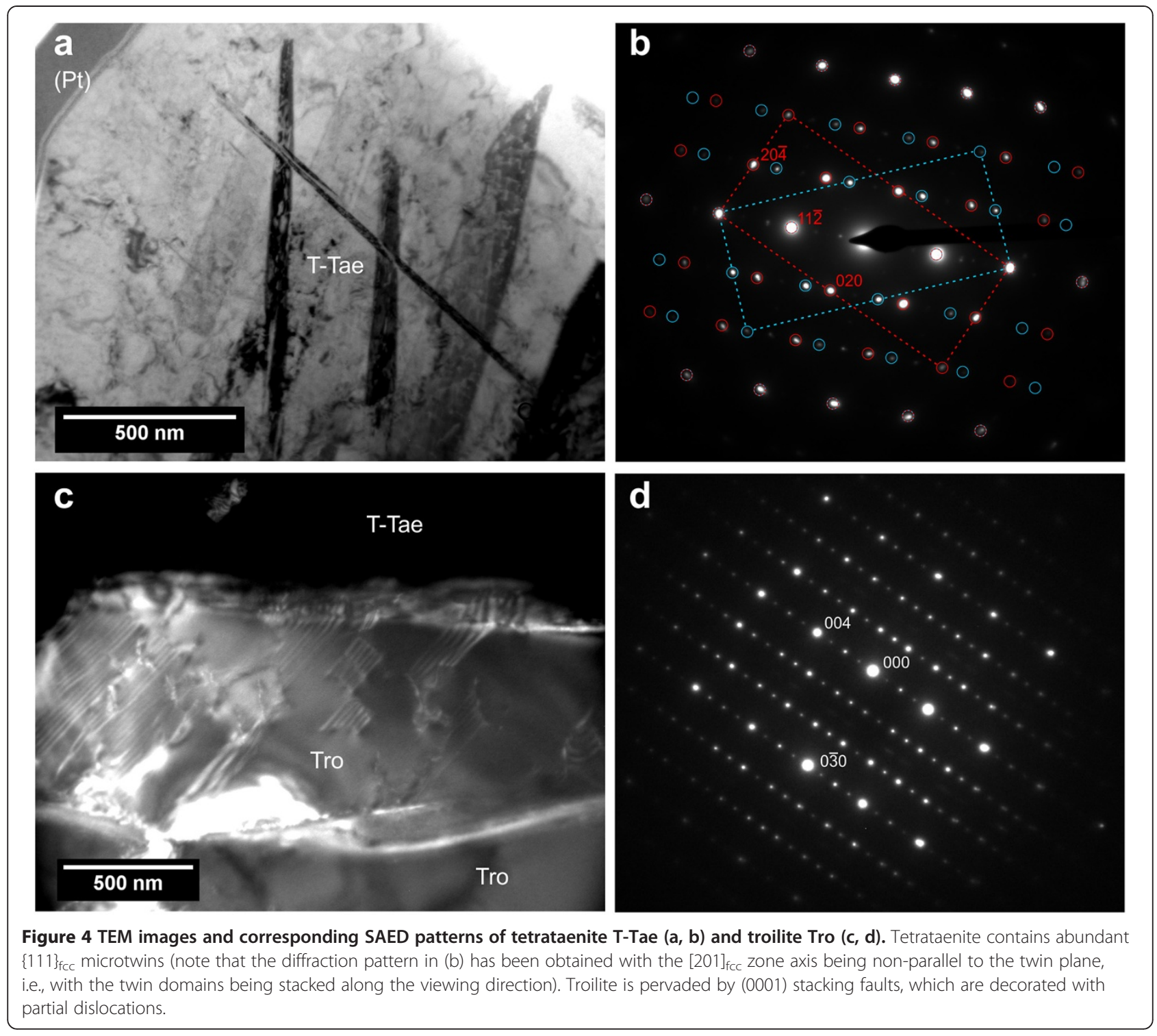

The chemical homogeneity of minerals suggests a high degree of thermal equilibration of particle RB-QD04-0042. In particular, the absence of $\mathrm{Ni}$ in troilite combined with the high $\mathrm{Ni}$ content in metal and the formation of the ordered tetrataenite structure allow us to better assess the thermal evolution of RB-QD04-0042. According to a recent study on the phase relations in the system Fe-Ni-S (Kitakaze et al. 2011), iron monosulfide coexisting with $\mathrm{Ni}$-rich taenite still contains $>1 \%$ to $2 \% \mathrm{Ni}$ in the investigated temperature range from $650^{\circ} \mathrm{C}$ to $450^{\circ} \mathrm{C}$. The Nipoor troilite observed in the Hayabusa particle must thus have equilibrated with $\mathrm{Ni}$-rich taenite at distinctly lower temperature. The observation of the ordered tetrataenite structure implies that $\mathrm{Fe}-\mathrm{Ni}$ exchange was locally still possible at temperatures below the critical ordering temperature of $320^{\circ} \mathrm{C}$ (Scott and Clarke 1979; Clarke and Scott 1980; Uehara et al. 2011).

\section{Evidence for shock metamorphism and collisional fragmentation}

The lattice defects found in minerals constituting particle RB-QD04-0042 are largely known as diagnostic shock indicators, in particular the planar fractures and [001] screw dislocations in olivine and also the $\{101\}$ microtwins in tetragonal tetrataenite, and the (0001) stacking faults in troilite (Figures 3 and 4). The particle was thus definitely involved in a collisional event. The shock event occurred probably well after the thermal metamorphism that equilibrated the studied regolith grain, because mechanical twinning in iron-based alloys is commonly known to be a low-temperature phenomenon. The heterogeneous distribution of fractures and dislocations in olivine, which are almost completely concentrated in subsample RB-QD04-0042-02, records a high stress gradient across the particle. 
Similar defect microstructures with planar fractures and [001] screw dislocations were reproduced in olivine by controlled laser shock experiments with nanosecond pulses (Langenhorst et al. 1999). Planar fractures and dislocations emitted from these fractures were both interpreted to have formed and propagated during the compressional phase of the shock wave. Therefore, a dislocation velocity could be estimated in these experiments using the length of the shock pulse and the distance of dislocations from the fracture planes. This velocity turned out to be on average 1 $\mathrm{km} \mathrm{s}^{-1}$ (Langenhorst et al. 1999). The penetration depth of dislocations in RB-QD04-0042 can be roughly estimated to be on the order of a few micrometers (up to about $6 \mu \mathrm{m}$ ). Taking into account the abovementioned average dislocation velocity of $1 \mathrm{~km} \mathrm{~s}^{-1}$, one can calculate a shock duration of a few nanoseconds (most probably less than $10 \mathrm{~ns}$ ). Therefore, the Hayabusa particle RBQD04-0042 was affected by a small-scale impact event. The fractured concave surface on one side of the particle suggests that either material was spalled off the surface, possibly by the collision with another regolith grain, or that RB-QD04-0042 itself is a spallation fragment of a larger particle which was partially or totally disrupted by hypervelocity collision. As a consequence of spallation, the sulfide-metal aggregate became probably exposed to the surface (Figure 1a). Thus, our particle provides evidence for a typical impact scenario on an asteroid, i.e., collisional fragmentation of its surface resulting in regolith formation (Melosh 1996; Housen and Holsapple 2003; Cintala and Hörz 2008).

\section{Conclusions}

This study reports the detailed investigation of a precious Hayabusa dust particle (RB-QD04-0042) from asteroid Itokawa using sophisticated SEM-FIB preparation and subsequent TEM characterization. The results of this combined methodological approach allow us to draw the following main conclusions:

1. The mineral compositions of olivine $\left(\mathrm{Fo}_{69} \mathrm{Fa}_{31}\right)$ and diopside $\left(\mathrm{En}_{48} \mathrm{Wo}_{42} \mathrm{Fs}_{10}\right)$ and the high Ni content in a troilite-tetrataenite assemblage are in agreement with the classification of the Hayabusa particle as $L L$-type ordinary chondrite material (Nakamura et al. 2011, 2012; Tsuchiyama 2014).

2. The obvious absence of $\mathrm{Ni}$ in troilite and the ordering of the FeNi alloy to form the tetrataenite structure suggest slow cooling and chemical equilibration at temperatures even below $320^{\circ} \mathrm{C}$ (i.e., transition temperature for $\mathrm{Fe}, \mathrm{Ni}$ ordering).

3. Despite the absence of visible impact craters on the surface of RB-QD04-0042, the dust particle is heavily fractured on one side, and only below this surface, distinct signs of shock metamorphism were detected by TEM. Based on the penetration depth of [001] screw dislocations in olivine and their propagation velocity, we estimate the pulse duration of the shock wave to be less than $10 \mathrm{~ns}$, indicating that the particle was involved in a small-scale impact. The fractured concave surface and the exposure of a sulfide-metal aggregate on one side of the regolith particle suggest that either material was spalled off the surface or that the particle represents a spallation fragment resulting from the collisional fragmentation of a larger regolith particle.

4. This study exemplifies that the combined use of FIB preparation and TEM characterization is an ideal tool for the three-dimensional site-specific examination of limited small dust particles returned by space missions. FIB preparation has two major advantages over ultramicrotomy. Cracking of samples, as is caused by ultramicrotome preparation (Jacob et al. 2009), does not develop in FIB preparation, and mineral assemblages are preserved in their original textural setting. In comparison to X-ray microtomography (Tsuchiyama et al. 2013), the strength of the FIB-TEM approach is its superior lateral resolution.

\section{Competing interests}

The authors declare that they have no competing interests.

\section{Authors' contributions}

$\mathrm{FL}$ and $\mathrm{DH}$ carried out and evaluated the analytical scanning and transmission electron microscopic investigations. DH produced TEM foils by focused ion beam preparation. FL wrote the manuscript with significant contributions by DH. KP and PVA conceived the study and helped to improve the manuscript. All authors read and approved the final manuscript.

\section{Acknowledgements}

We are very grateful to JAXA for providing access to the precious Hayabusa particles and for providing financial support for the attendance of the 1st Hayabusa symposium. The research leading to these results has received funding from the Deutsche Forschungsgemeinschaft (LA 830/14-1 to FL) and the European Union Seventh Framework Programme (FP7/2007-2013) under grant agreement no. 312483 (ESTEEM2). We thank five anonymous reviewers for their constructive suggestions.

\section{Author details}

${ }^{1}$ Analytical Mineralogy of Micro- and Nanostructures, Institute of Geoscience, Friedrich Schiller University Jena, Carl-Zeiss-Promenade 10, D-07745 Jena, Germany. ${ }^{2}$ Max Planck Institute for Intelligent Systems, Heisenbergstr. 3, D-70569 Stuttgart, Germany.

Received: 28 February 2014 Accepted: 4 September 2014 Published: 17 September 2014

\section{References}

Abe M, Yada T, Okada T, Uesugi M, Karouji Y, Ishibashi Y, Yakame S, Mukai T, Fujimura A, Fujimoto M (2012) Present status and future plans for international announcement of opportunity for research of the Hayabusa returned particles. Meteorit Planet Sci 47:A35

Brearley AJ, Jones RH (1998) Chondritic meteorites. In: Papike JJ (ed) Planetary materials, vol 36, Reviews in mineralogy. Mineralogical Society of America, Washington, pp 3-370

Cintala MJ, Hörz F (2008) Experimental impacts into chondritic targets. Part I: disruption of an L6 chondrite by multiple impacts. Meteorit Planet Sci 43(4):771-803 
Clarke RS, Scott ERD (1980) Tetrataenite - ordered FeNi, a new mineral in meteorites. Am Mineral 65:624-630

Harries D, Langenhorst F (2014) The mineralogy and space weathering of a regolith grain from 25143 Itokawa and the possibility of annealed solar wind damage., Earth, Planets and Space. this volume

Harries D, Pollok K, Langenhorst F (2011) Translation interface modulation in NC-pyrrhotites: direct imaging by TEM and a model toward understanding partially disordered structural states. Am Mineral 96(5-6):716-731

Housen KR, Holsapple KA (2003) Impact cratering on porous asteroids. Icarus 163:102-119

Jacob D, Stodolna J, Leroux H, Langenhorst F, Houdellier F (2009) Pyroxenes microstructure in comet 81P/Wild 2 terminal Stardust particles. Meteorit Planet Sci 44(10):1475-1488

Jarosevich E (1990) Chemical analyses of meteorites: a compilation of stony and iron meteorite analyses. Meteoritics 25:323-337

Kerr RA (2011) Hayabusa gets to the bottom of deceptive asteroid cloaking. Science 333:1081

Kitakaze A, Sugaki A, Itoh $\mathrm{H}$, Komatsu $\mathrm{R}$ (2011) A revision of phase relations in the system Fe-Ni-S from $650^{\circ}$ to $450^{\circ} \mathrm{C}$. Can Mineral 49:1687-1710

Langenhorst F, Joreau P, Doukhan JC (1995) Thermal and shock metamorphism of the Tenham chondrite: a TEM examination. Geochim Cosmochim Acta 59(9):1835-1845

Langenhorst F, Boustie M, Migault A, Romain JP (1999) Laser shock experiments with nanoseconds pulses: a new tool for the reproduction of shock defects in olivine. Earth Planet Sci Lett 173(3):333-342

Langenhorst F, Poirier JP, Deutsch A, Hornemann U (2002) Experimental approach to generate shock veins in single crystal olivine by shear melting. Meteorit Planet Sci 37(11):1541-1553

Leroux H (2001) Microstructural shock signatures of major minerals in meteorites. Eur J Mineral 13(2):253-272

Leroux H, Doukhan JC, Langenhorst F (1994) Microstructural defects in experimentally shocked diopside - a TEM characterization. Phys Chem Miner 20(8):521-530

Mang C, Kontny A, Harries D, Langenhorst F, Hecht L (2012) Iron deficiency in pyrrhotite of suevites from the Chesapeake Bay impact crater, USA - a consequence of shock metamorphism? Meteorit Planet Sci 47(2):277-295

Melosh HJ (1996) Oxford monographs on geology and geophysics, vol 11. Oxford University Press Inc, Oxford, p 245

Nakamura T, Noguchi T, Tanaka M, Zolensky ME, Kimura M, Tsuchiyama A, Nakato A, Ogami T, Ishida H, Uesugi M, Yada T, Shirai K, Fujimura A, Okazaki R, Sandford SA, Ishibashi Y, Abe M, Okada T, Ueno M, Mukai T, Yoshikawa M, Kawaguchi J (2011) Itokawa dust particles: a direct link between S-type asteroids and ordinary chondrites. Science 333:1113-1116

Nakamura E, Makishima A, Moriguti T, Kobayashi K, Tanaka R, Kunihiro T, Tsujimori T, Sakaguchi C, Kitagawa H, Ota T, Yachi Y, Yada T, Abe M, Fujimura A, Ueno M, Mukai T, Yoshikawa M, Kawaguchi J (2012) Space environment of an asteroid preserved on micrograins returned by the Hayabusa spacecraft. Proc Natl Acad Sci 109:E624-E629

Nakashima D, Kita NT, Ushikubo T, Noguchi T, Nakamura T, Valley JW (2013) Oxygen three-isotope ratios of silicate particles returned from asteroid Itokawa by the Hayabusa spacecraft: a strong link with equilibrated LL chondrites. Earth Planet Sci Lett 379:127-136

Noguchi T, Nakamura T, Kimura M, Zolensky ME, Tanaka M, Hashimoto T, Konno M, Nakato A, Ogami T, Fujimura A, Abe M, Yada T, Mukai T, Ueno M, Okada T, Shirai K, Ishibashi Y, Okazaki R (2011) Incipient space weathering observed on the surface of Itokawa dust particles. Science 333:1121-1125

Scott ERD, Clarke RS (1979) Identification of clear taenite in meteorites as ordered FeNi. Nature 281(5730):360-362

Tsuchiyama A (2014) Asteroid Itokawa - a source of ordinary chondrites and a laboratory for surface processes. Elements 10(1):45-50

Tsuchiyama A, Nakano T, Uesugi K, Uesugi M, Takeuchi A, Suzuki Y, Noguchi R, Matsumoto T, Matsuno J, Nagano T, Imai Y, Nakamura T, Ogami T, Noguchi T, Abe M, Yada T, Fujimura A (2013) Analytical dual-energy microtomography: a new method for obtaining three-dimensional mineral phase images and its application to Hayabusa samples. Geochim Cosmochim Acta 116:5-16

Uehara M, Gattacceca J, Leroux H, Jacob D, van der Beek CJ (2011) Magnetic microstructures of metal grains in equilibrated ordinary chondrites and implications for paleomagnetism of meteorites. Earth Planet Sci Lett 306:241-252

Van Cappellen E, Doukhan JC (1994) Quantitative X-ray microanalysis of ionic compounds. Ultramiscroscopy 53:343-349
Yurimoto H, Abe K, Abe M, Ebihara M, Fujimura A, Hashiguchi M, Hashizume K, Ireland TR, Itoh S, Katayama J, Kato C, Kawaguchi J, Kawasaki N, Kitajima F, Kobayashi S, Meike T, Mukai T, Nagao K, Nakamura T, Naraoka H, Noguchi T, Okazaki R, Park C, Sakamoto N, Seto Y, Takei M, Tsuchiyama A, Uesugi M, Wakaki S, Yada T et al (2011) Oxygen isotopic compositions of asteroidal materials returned from Itokawa by the Hayabusa mission. Science 333:1116-1119

doi:10.1186/1880-5981-66-118

Cite this article as: Langenhorst et al:: Mineralogy and defect microstructure of an olivine-dominated Itokawa dust particle: evidence for shock metamorphism, collisional fragmentation, and LL chondrite origin. Earth, Planets and Space 2014 66:118.

\section{Submit your manuscript to a SpringerOpen ${ }^{\odot}$ journal and benefit from:}

- Convenient online submission

- Rigorous peer review

- Immediate publication on acceptance

- Open access: articles freely available online

- High visibility within the field

- Retaining the copyright to your article

Submit your next manuscript at $>$ springeropen.com 